\title{
Malar Relocation with Reverse-L Osteotomy and Autogenous Bone Graft
}

\author{
Se Hoon Yoon ${ }^{1}$, \\ Euicheol Jeong ${ }^{2}$, \\ Jee Hyeok Chung ${ }^{3}$ \\ ${ }^{1}$ Department of Plastic and Reconstructive \\ Surgery, Seoul National University College of \\ Medicine, Seoul; \\ ${ }^{2}$ Department of Plastic Surgery, SMG-SNU \\ Boramae Medical Center, Seoul; \\ ${ }^{3}$ Department of Plastic Surgery, Myongi \\ Hospital, Goyang, Korea
}

The zygomaticomaxillary complex (ZMC) functions as a buttress for the face and is the cornerstone to a person's aesthetic appearance, by both setting the midfacial width and providing prominence to the cheek. Malar deficiency is often acquired by blunt injury incurred in a traumatic accident, resulting in ZMC fracture. A 48-year-old male patient presented a right ZMC fracture after contusion injury by a baseball. He only received conservative management and later he suffered discomfort during mouth opening at the moment of mastication, due to trismus involving the temporomandibular joint. In the current case, we describe a surgical technique, by which the malar body is shifted anteriorly and laterally after combined oblique-vertical osteotomy. The technique presented, eventually restored the former aesthetic position of the malar complex and symmetry, and, moreover, improved mastication function

Keywords: Zyomatic fractures / Malunited fractures

\section{INTRODUCTION}

The zygomaticomaxillary complex (ZMC) functions as a buttress for the face and is the cornerstone to a person's aesthetic appearance, by both setting the midfacial width and providing prominence to the cheek [1]. The ZMC comprises the zygomatic arch, inferior orbital rim, the anterior and posterior maxillary sinus walls, and the lateral orbital rim. This structural complexity makes it difficult to set an ideal projection. Malar deficiency is often acquired by blunt injury incurred in a traumatic accident, which results in high-energy ZMC fracture and causes inferior, medial and posterior displacement. Generalized midface deficiency can occur, giving rise to a disproportionate face and a masticatory functional deficit, by inward displacement of the zygo-

Correspondence: Euicheol Jeong

Department of Plastic Surgery, SMG-SNU Boramae Medical Center, 20 Boramae-ro 5 Gil, Dongjak-gu, Seoul 07061, Korea

E-mail: ecjeong@snu.ac.kr

Received Jul 31, 2017 / Revised Oct 18, 2017 / Accepted Nov 2, 2017 matic arch.

In the current case, we describe a surgical technique, by which the malar body is shifted anteriorly and laterally after combined oblique-vertical osteotomy. The technique presented, eventually restored the former aesthetic position of the malar complex and symmetry, and, moreover, improved mastication function.

\section{CASE REPORT}

A 48-year-old male patient presented a right ZMC fracture after contusion injury by a baseball. At the time of the accident, he did not receive any surgical correction of the malpositioned zygoma, but only conservative management. For the past 7 years, he had suffered discomfort during mouth opening at the moment of mastication, due to trismus involving the temporomandibular joint. The patient visited the hospital, hoping to restore the original functional status and correct depressed malar prominence.

At facial computed tomography (CT) evaluation, he had inferior, posterior and medial displacement of the right malar complex 
with malunion at the zygomatic arch (Fig. 1). He had no enophthalmos nor functional discomfort such as entropion.

Incision was made about $5 \mathrm{~mm}$ above the mucogingival junction from the edge of lateral incisor to second molar tooth. After horizontal incision of the periosteum, subperiosteal dissection was extended superiorly over the malar eminence just below infraorbital rim and laterally at the zygomatic arch to the region of the zygomaticotemporal suture. After exposure of the malar bone, a further dissection was done under precaution to identify the infraorbital foramen and zygomaticomaxillary foramen. Although, subciliary incision was considered preoperatively, it was unnecessary since infraorbital malar area was sufficiently exposed only with intraoral incision (Fig. 2). Reverse-L osteotomy (com-
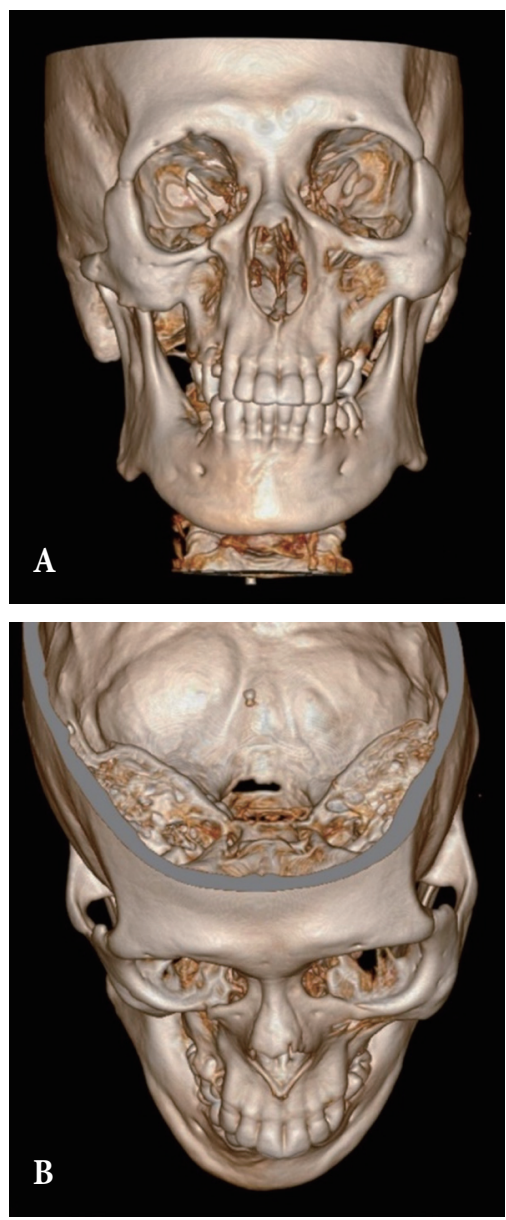

Fig. 1. (A, B) Preoperative computed tomography image. Compared to the unaffected left side, the zygomaticomaxillary complex of the right face presented inferior, medial and posterior displacement.

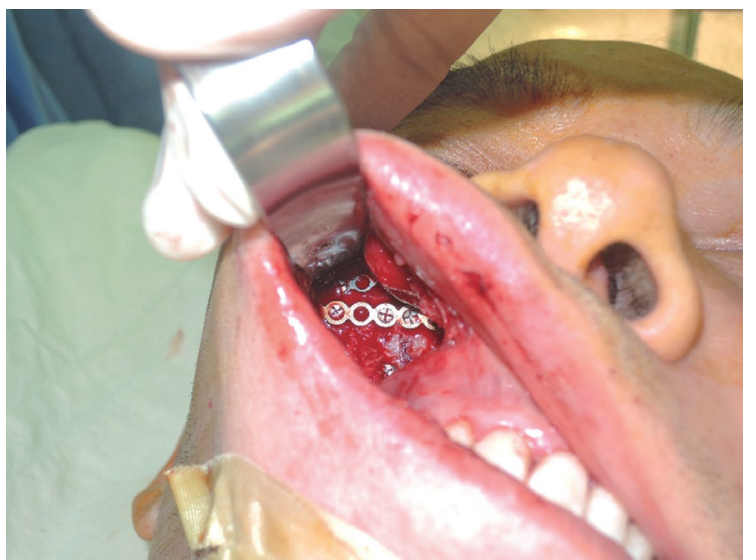

Fig. 2. Intraoperative photo image showing retractor device inserted at the intraoral incision site. Wide subperiosteal dissection was possible over the malar eminence with no need of subciliary incision.

bined vertical and horizontal osteotomy) was performed using a reciprocating saw along the zygomatic process of the maxilla (Fig. 3) under protection of previously explored neurovascular bundle with retractors. The vertical osteotomy passed through the anterior and posterior wall of the maxillary sinus, lateral to the infraorbital foramen, and horizontal osteotomy cuts were made about 3 $\mathrm{mm}$ below the zygomaticomaxillary foramen. Using Gilles' incision, a dissection under the deep temporal fascia allowed access to the malunioned zygomatic arch. Through this plane, a blind osteotomy was done with an oscillating saw, from the inner surface of the malunioned bone segment and movement of the lateral segment of the zygoma was eased.

The lateral segment of the malar bone was pulled anterolaterally, for malar augmentation. The bony gap formation between the medial and lateral segment of the malar bone, was filled in a wedge fashion with iliac bone $(20 \times 10 \times 5 \mathrm{~mm}$, carved into a rectangular shape) harvested from the right anterosuperior iliac spine.

Bone segments were fixed, using a nonabsorbable rigid titanium plate and screws (1.7 mm midface standard plates, Stryker) at the anterior maxillary surface (Fig. 4). However, there was no fixation performed at the zygomatic arch osteotomy site, leaving the arch free.

The patient showed no signs of complications, such as hematoma, wound dehiscence, or sensory loss. A postoperative CT image was obtained on the day of surgery and the patient was discharged 
A

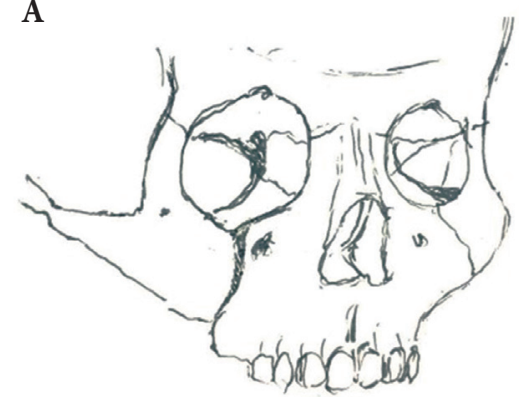

B

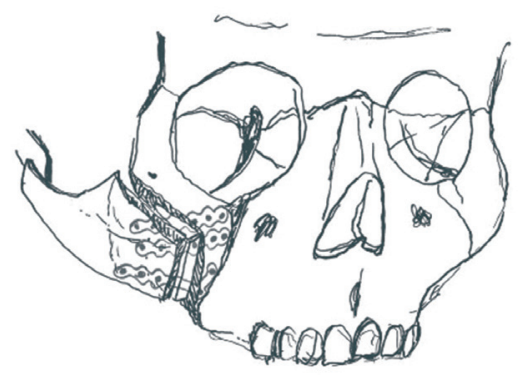

Fig. 3. (A, B) Reverse-L osteotomy (combined horizontal and vertical osteotomy) and blind osteotomy at the zygomatic arch results in freeing of the lateral malar bone segment.

a week later. Three months after surgery, a follow-up CT image showed the fixed bone segments had maintained the immediate postoperative shape. There was no sign of relapse of the zygomatic depression, nor sign of compression of the zygomatic arch to the temporalis muscle (Fig. 5). Facial asymmetry was much improved by restoration of the vertical height of the lateral bony projection (Fig. 6). The right iliac crest donor site healed spontaneously and the bone segments recovered, ultimately leaving no bony defect.

\section{DISCUSSION}

The malar eminence is an important component of facial aesthetics, contributing to a youthful facial appearance. Flatness and a relatively inferior position of the malar eminence, generates an older, expressionless and depressed profile to the face. Usually, the supraorbital ridge should project $4-8 \mathrm{~mm}$ anterior to the anterior surface of the cornea, and the most prominent part of the malar eminence should project slightly beyond the cornea [2]. When the malar-zygomatic structure collapses, secondary to a trauma inci-
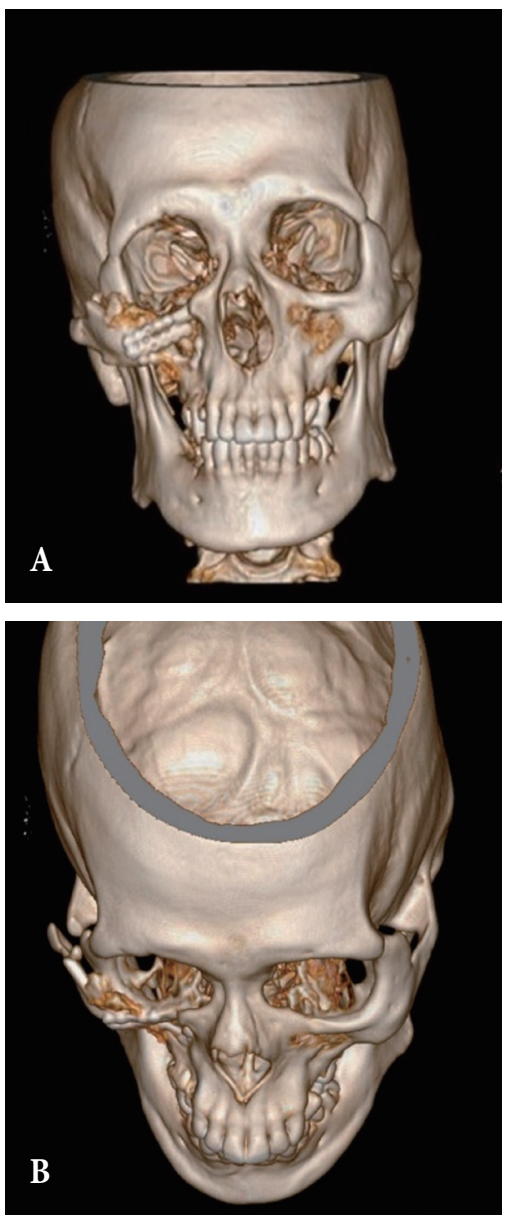

Fig. 4. (A, B) Rigid fixation was held using three $1.7 \mathrm{~mm}$ nonabsorbable titanium plate and screws, and stability was sustained until 6 months, postoperatively.

dent, the midface may seem hypoplastic and facial proportion, harmony and symmetry, might be severely disrupted. In order to correct this acquired deformity, surgical treatment, such as Le Fort osteotomies, malar bone osteotomies, autogenous onlay bone or cartilage grafts, and malar augmentation using a prosthesis, have been attempted [3]. However, alloplastic materials have complications, like host reaction, migration, and displacement of the implant, causing unaesthetic and asymmetric outcomes [4]. Also, implants may become obvious with time as the overlying soft tissues atrophy and sag. The capsule formation that accompanies smooth-surfaced implants, further exaggerates the tendency towards implant visibility [5].

Osteotomy and reduction can be done solely without bone 

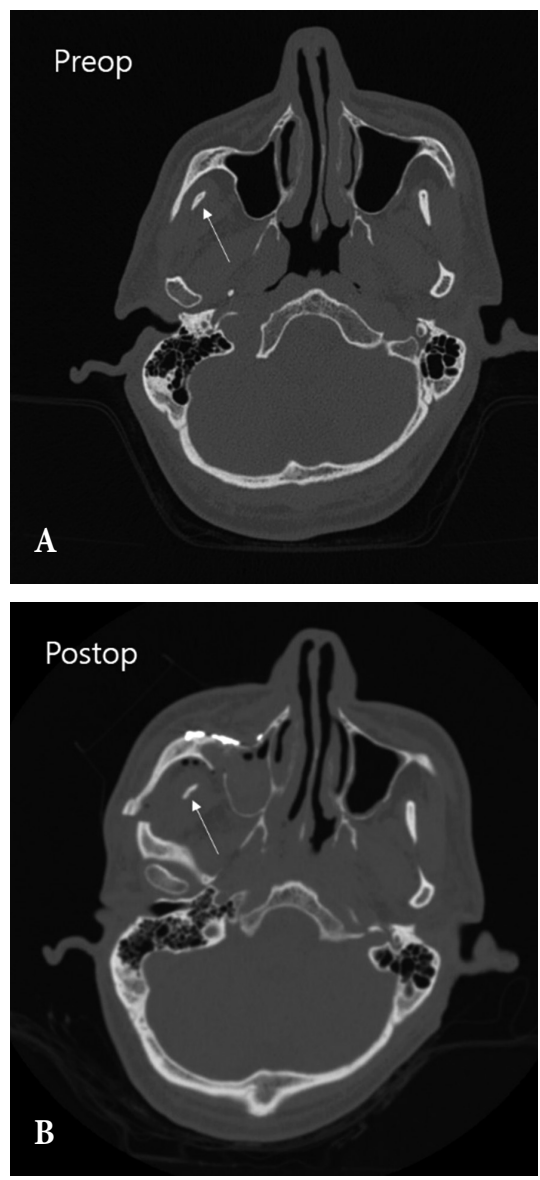

Fig. 5. Preoperative (A) and postoperative (B) axial computed tomography image of the patient. The white arrow indicates the coronoid process, and there is a expansion of the space between the arch and the process, postoperatively.

grafting, but this can lead to reoperation due to instability. It has been reported that osteotomy is useful up to 4 months postinjury, but after that its instability requires bone grafting since bone remodeling has occurred already [6], making it difficult to recover the bone segment to its original position. For thorough correction, multiple osteotomy and bone graft can be performed but this requires invasive procedures, such as additional coronal incision to expose the whole ZMC surface, which was declined by the patient.

Since there was no functional nor aesthetic discomfort after malunion at the infraorbital rim, surgical correction was not considered. On the other hand, the patient complained about facial asymmetry due to malar prominence deficiency, which was the
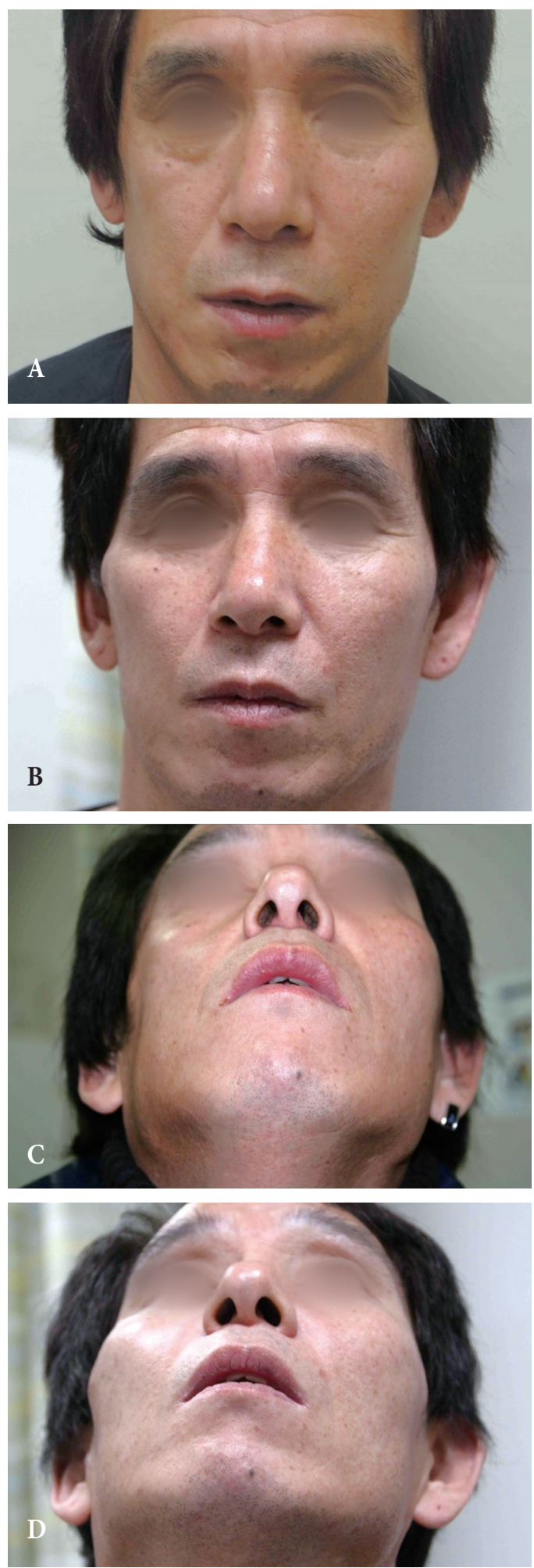

Fig. 6. Preoperative $(A, C)$ and postoperative $(B, D)$ photo of the patient. The zygomaticomaxillary complex recovered its symmetry resulting in a good aesthetic outcome. 
key factor of our operative planning.

Although cancellous bone graft has resorption issues at longterm follow-up [7], out-fracturing of the zygoma in this instance, the breadth of space obtained allowed bone transport through the grafted bone, with an intact periosteum covering the outer surface. So, regardless of the bone resorption or defect between the osteotomized surface, the interposition bone graft works as a scaffold for bone regeneration ensuring gradual ossification [8]. At 6 months postoperative, the patient's CT image showed the cortical bone portion was well preserved, maintaining the stability of the titanium locking plate and much of the cancellous bone seemed to be resorbed and replaced with new bone. This method is similar to the commonly used method during tibial Ilizarov surgery [9], which uses a cancellous bone graft at the gap at the distraction osteogenesis site.

The instability of the zygomatic arch was not an issue, as the zygomatic arch is not the key structure supporting the bony complex in the malar region. The zygomatic arch is a relatively weak bone because it is laterally positioned and is a combination of the zygoma and temporal bone. For multiple combined fractures of the zygoma bone with arch displacement, fixation of the arch after reduction is not always necessary, after sufficient stability of the bony complex has been accomplished [10]. This justifies plate fixation solely in the malar region, leaving the osteotomized zygomatic arch without any additional support. In addition, the unfixed zygomatic arch enables the distal segment to acquire a natural position by free movement after early mastication. This seems to prevent long complications of mastication issues, which can occur by restriction of the temporalis muscle or coronoid process movement by a malpositioned arch.

Though vertical osteotomy alone, can move the lateral segment of the zygoma in the desired direction, combined horizontal and vertical osteotomy enables a larger lateral zygomatic segment to be freed from the complex, for stable fixation. Also, the infraorbital foramen and zygomaticomaxillary foramen, was taken from the upper and medial border of the osteotomy, which does not jeopardize injury to the neurovascular pedicle.

In old tripod fracture patients, depression of the malar eminence can cause detrimental results of facial aesthetics, due to a loss of both, the symmetry and harmony of facial proportions. Even when the reduction is too late, malar augmentation by reverse-L osteotomy and autologous bone interposition graft, can help recover normal bone structure. This is a simple and safe surgical technique, with both functional and cosmetic gain outcomes.

\section{CONFLICT OF INTEREST}

No potential conflict of interest relevant to this article was reported.

\section{PATIENT CONSENT}

The patient provided written informed consent for the publication and the use of their images.

\section{REFERENCES}

1. Lee EI, Mohan K, Koshy JC, Hollier LH Jr. Optimizing the surgical management of zygomaticomaxillary complex fractures. Semin Plast Surg 2010;24:389-97.

2. Bell WH. Malar midfacial augmentation. In: Bell WH, editor. Modern practice in orthognathic and reconstructive surgery. Philadelphia: Saunders; 1992.p.2289-97.

3. Salyer KE. Malar augmentation using lamellar split osteotomy. J Craniofac Surg 1994;5:116-23.

4. Jensen J, Reiche-Fischel O, Sindet-Pedersen S. Autogenous mandibular bone grafts for malar augmentation. J Oral Maxillofac Surg 1995; 53:88-90

5. Neligan P, Warren RJ, Van Beek A. Plastic surgery. London: Elsevier Saunders; 2013.

6. Carr RM, Mathog RH. Early and delayed repair of orbitozygomatic complex fractures. J Oral Maxillofac Surg 1997;55:253-8.

7. Zins JE, Whitaker LA. Membranous versus endochondral bone: implications for craniofacial reconstruction. Plast Reconstr Surg 1983;72: 778-85.

8. Mommaerts MY, Abeloos JV, De Clercq CA, Neyt LF. The 'sandwich' zygomatic osteotomy: technique, indications and clinical results. J Craniomaxillofac Surg 1995;23:12-9.

9. Mukhopadhaya J, Raj M. Distraction osteogenesis using combined locking plate and Ilizarov fixator in the treatment of bone defect: a report of 2 cases. Indian J Orthop 2017;51:222-8.

10. Fonseca RJ, Walker RV, Betts NJ, Barber HD. Oral and maxillofacial surgery. 2nd ed. Philadelphia: Saunders; 1997. 